\title{
Etude de la teneur en éléments minéraux des produits obtenus lors de l'ultrafiltration du lait sur membrane
}

\author{
par \\ G. BRULE, J. L. MAUBOIS et J. FAUQUANT \\ Laboratoire de Recherches de Technologie Laitière I.N.R.A. \\ 65, rue de Saint-Brieuc - 35042 Rennes cedex
}

On sait, depuis les travaux de Guittonneau et Chevalier (1935), qu'un type de fromage peut être caractérisé, entre autres choses, par le rapport existant entre sa teneur en calcium et sa teneur en phosphore. La composition minérale des fromages exerce dans son ensemble une influence essentielle sur les qualités organoleptiques de ces produits, notamment sur leur texture (Veisseyre, 1966). De faibles variations de la teneur en minéraux des coagulum obtenus après action de la présure sur le lait peuvent entraîner des modifications considérables des qualités organoleptiques des fromages (Davies et White, 1960). Or la teneur en minéraux des fromages résulte de la répartition de ces mêmes minéraux, provenant du lait, entre le lactosérum et le coagulum au cours de l'égouttage, répartition dépendant étroitement de l'état physico-chimique dans lequel se trouvent ces éléments pendant la synérèse.

Le remplacement de cette synérèse par l'ultrafiltration sur membrane du lait destiné à la transformation en fromage (Maubois et Mocquot, 1971) nécessite que soient bien connus non seulement la composition minérale de chacun des liquides obtenus de part et d'autre de la membrane (rétentat et perméat) mais aussi les facteurs qui peuvent influencer cette répartition.

Dans leur étude sur la phase aqueuse du lait, Davies et White (1960) ont montré que la composition minérale des ultrafiltrats obtenus par mise en contact de lait écrémé avec une membrane de cellophane ou de cellulose Visking fixée dans un appareillage expérimental " ad hoc " pouvait varier légèrement selon la nature de la membrane, l'intensité de la pression et la température du lait.

Dans une étude récente, Pompei et al. (1973) ont mis en évidence une rétention des constituants minéraux solubles du lait lorsqu'on 
ultrafiltre à basse température $\left(5^{\circ} \mathrm{C}\right)$. Par contre, Madsen (1974) n'a pas observé cette rétention des minéraux solubles ( $\mathrm{Na}-\mathrm{K}$ ) au cours d'ultrafiltrations réalisées dans les conditions de température et de concentration indiquées par Maubois (1973).

L'objet du présent travail est d'étudier la répartition des principaux minéraux du lait $(\mathrm{Ca}-\mathrm{Mg}-\mathrm{K}-\mathrm{Na})$ entre le rétentat et le perméat au cours de l'ultrafiltration du lait et de déterminer l'influence que peuvent avoir sur cette répartition les caractéristiques de la membrane et de l'appareillage, le $\mathrm{pH}$ et enfin l'addition (conjuguée éventuellement avec un abaissement de $\mathrm{pH}$ ) de chlorure de sodium.

\section{Ultrafiltration}

\section{MATERIEL ET METHODES}

Les caractéristiques des membranes et des appareillages sont indiquées dans le tableau 1.

TABLEAU 1

Caractéristiques des membranes et des appareillages utilisés

\begin{tabular}{|c|c|c|}
\hline Appareillage & Membrane & $\begin{array}{c}\text { Surface membranaire } \\
\text { de l'appareil }\end{array}$ \\
\hline $\begin{array}{l}\text { Romicon, type hollow } \\
\text { fiber }\end{array}$ & $\begin{array}{l}\text { PM } 30 \\
\text { XM } 50\end{array}$ & $\begin{array}{ll}1 & \mathrm{~m}^{2} \\
1 & \mathrm{~m}^{2}\end{array}$ \\
\hline \multirow{2}{*}{ D.D.S. } & $\begin{array}{l}600 \\
\text { AR6 }\end{array}$ & $\begin{array}{l}0,5 \mathrm{~m}^{2} \\
0,5 \mathrm{~m}^{2}\end{array}$ \\
\hline & $\begin{array}{l}600 \\
\text { AR6 }\end{array}$ & $\begin{array}{ll}18 \mathrm{~m}^{2} \\
18 & \mathrm{~m}^{2}\end{array}$ \\
\hline Rhône-Poulenc & Iris 3042 & $11 \mathrm{~m}^{2}$ \\
\hline
\end{tabular}

Le lait était du lait écrémé de grand mélange pasteurisé à $72^{\circ} \mathrm{C}$ pendant $15 \mathrm{~s}$.

L'ultrafiltration était réalisée à $20^{\circ} \mathrm{C}$; sa durée n'excédait pas $2 \mathrm{~h}$, aucune variation de $\mathrm{pH}$ n'était constatée au cours de ce laps de temps.

Pour étudier l'influence respective du facteur de concentration (poids de lait/poids de rétentat) et $\mathrm{du} \mathrm{pH}$ du lait mis en œuvre, le lait était acidifié jusqu'au $\mathrm{pH}$ recherché avec une solution d'acide 
lactique $M$; des échantillons d'ultrafiltrat et de rétentat étaient prélevés à différents facteurs de concentration.

Pour étudier l'influence du $\mathrm{pH}$ sur la composition minérale des rétentats de concentrations différentes, l'ulrafiltration du lait était conduite jusqu'à l'obtention de la concentration désirée. Ensuite, de façon à maintenir la concentration constante, l'ultrafiltrat était mélangé avec le rétentat tandis que le $\mathrm{pH}$ de ce dernier était ajusté à des valeurs déterminées par addition d'acide lactique $\mathbf{M}$. On faisait en sorte que le volume des échantillons d'ultrafiltrat prélevés aux différentes valeurs du $\mathrm{pH}$ fut égal à celui de la solution lactique ajoutée afin de ne pas modifier le facteur de concentration.

\section{Déterminations analytiques}

La teneur en substance sèche des échantillons de lait, de rétentat et d'ultrafiltrat était déterminée par dessiccation à l'étuve à $102^{\circ} \mathrm{C}$ $105^{\circ} \mathrm{C}$.

La détermination des teneurs en substances minérales était réalisée par absorption atomique sur un appareil Techtron 1200 suivant le protocole de Murthy et Rheh (1967) légèrement modifié comme suit : environ $5 \mathrm{~g}$ d'échantillon étaient pesés puis dilués avec environ $200 \mathrm{ml}$ d'eau distillée acidifiée avec $10 \mathrm{ml}$ d'HCl N afin de favoriser la solubilisation des minéraux; la solution était complétée à $500 \mathrm{ml}$ avec de l'eau distillée. Cette solution mère était diluée 10 fois avec une solution d'un sel de lanthane (500 ppm La) pour le dosage du calcium et du magnésium et avec une solution d'un sel de césium (500 ppm Cs) pour le dosage du sodium et du potassium.

Le dosage du phosphore total était réalisé selon la méthode préconisée par la F.I.L. (norme no 33).

Les déterminations de $\mathrm{pH}$ étaient effectuées avec un appareil Tacussel Isis 4000 à $\pm 0,05$ unité $\mathrm{pH}$ près.

\section{RESULTATS}

\section{Influence de la nature de la membrane et du type d'appareillage}

Les résultats indiqués dans le tableau 2 montrent qu'avec les trois types d'appareillages et les différentes membranes expérimentées on n'observe pas d'influence sur les teneurs en $\mathrm{Ca}, \mathrm{Mg}, \mathrm{Na}$ et $\mathrm{K}$ des ultrafiltrats, aussi bien dans le cas d'ultrafiltrations effectuées à pH 6,6 que de celles effectuées à pH 6,0.

Ayant également vérifié qu'à un $\mathrm{pH}$ donné les teneurs en minéraux des ultrafiltrats obtenus à différents facteurs de concentration étaient indépendantes de la membrane et de l'appareillage, nous avons réalisé la suite de notre étude pour des raisons de commodité, 
TABLEAU 2. - Analyse minérale des ultrafiltrats totaux obtenus sur différents appareils

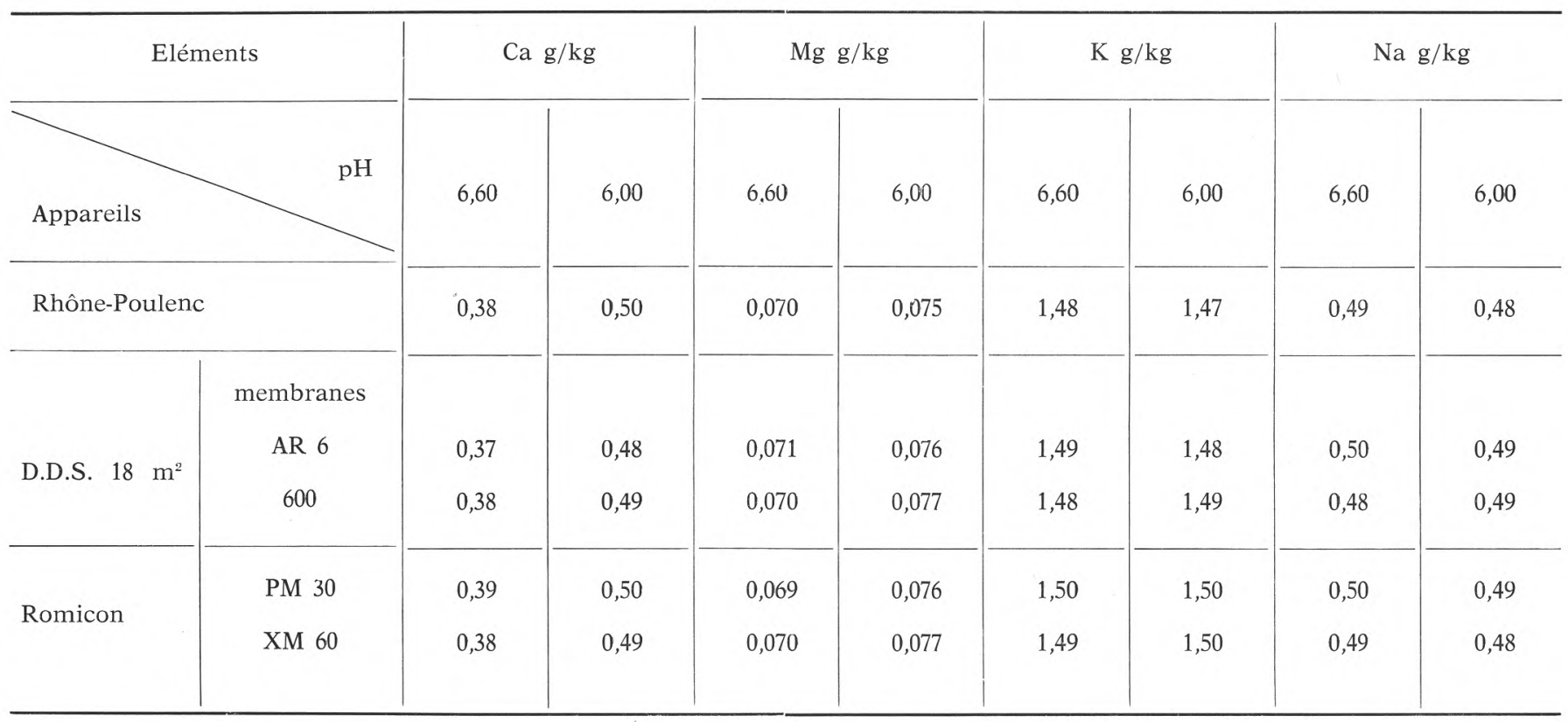




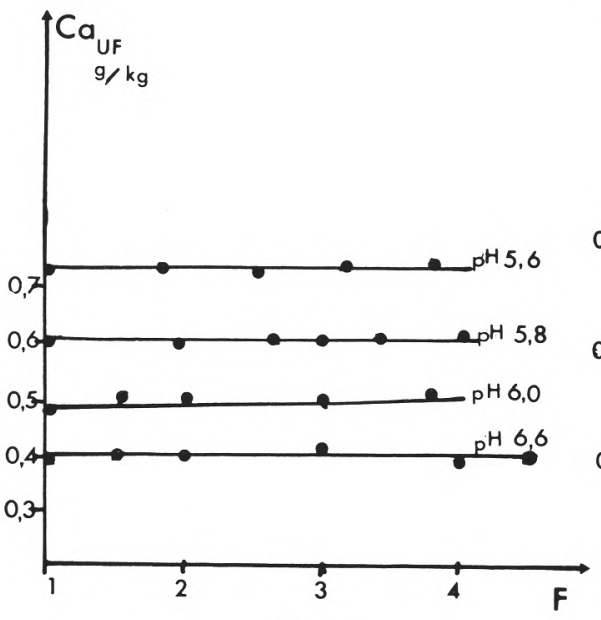

Fig 1

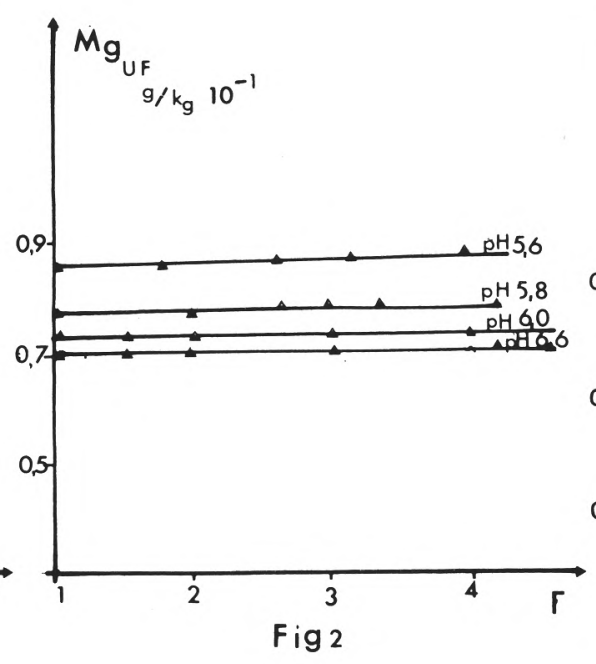

fig. $1-2-3$

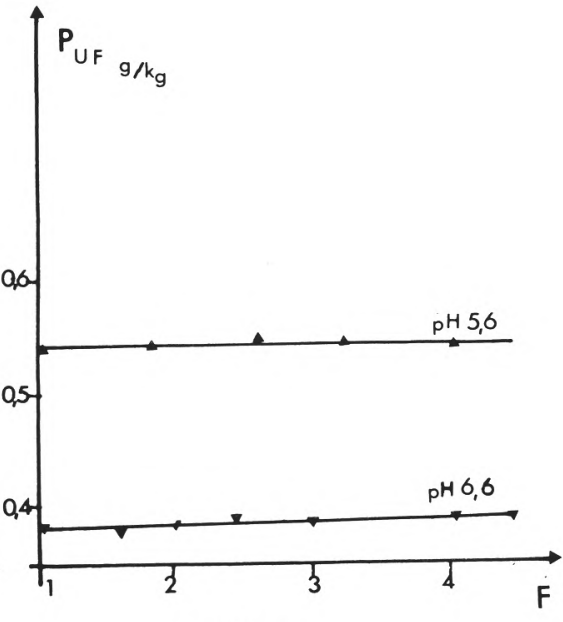

Fig 3

Variation, en fonction du facteur de concentration, de la teneur en calcium, magnésium et phosphore de l'ultrafiltrat obtenu par ultrafiltration de lait à différents $\mathrm{pH}$ 


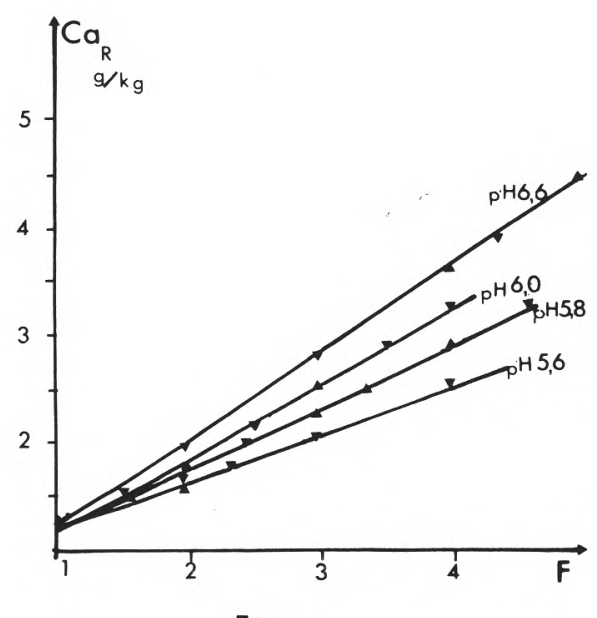

Fig 4

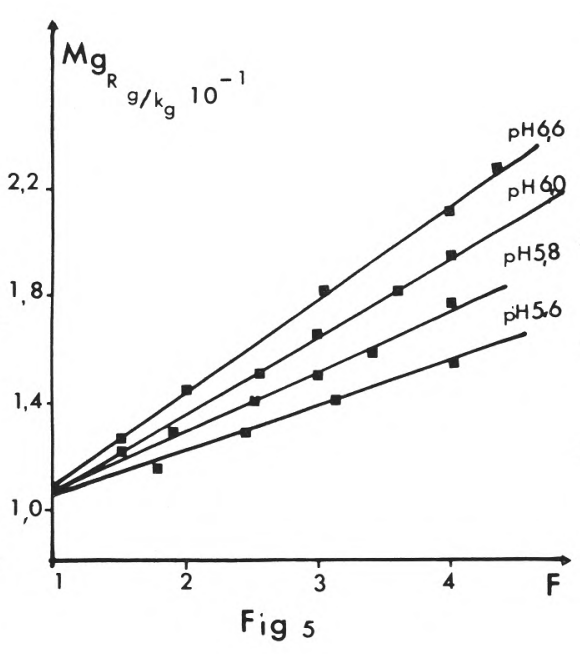

fig. $4-5-6$

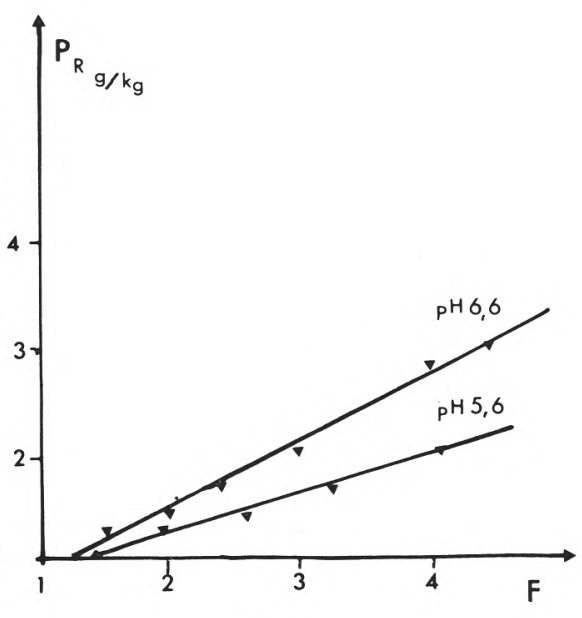

Fig 6

Variation, en fonction du facteur de concentration, de la teneur en calcium, magnésium et phosphore du rétentat obtenu par ultrafiltration de lait à différents $\mathrm{pH}$ 
sur un seul appareillage, le module Romicon, équipé d'une membrane XM 50.

\section{Influence du facteur de concentration sur la composition minérale des rétentats obtenus par ultrafiltration à différents $\mathrm{pH}$}

Les figures 1 - 2 et 3 indiquent que, pour un $\mathrm{pH}$ donné les teneurs en $\mathrm{Ca}-\mathrm{Mg}$ et $\mathrm{P}$ de l'ultrafiltrat demeurent constantes, quel que soit le facteur de concentration. Dans le tableau 3 nous avons reporté les teneurs de ces éléments dans l'ultrafiltrat aux différents $\mathrm{pH}$.

TABLEAU 3

Composition en minéraux des ultrafiltrats obtenus à différents $\mathrm{pH}$

\begin{tabular}{l|c|c|c}
\hline & $\mathrm{Ca} / \mathrm{kg}$ & $\mathrm{Mg} / \mathrm{kg}$ & $\mathrm{P}$ g/kg \\
\cline { 2 - 3 } & 1,25 & 0,100 & 1,00 \\
Lait & 0,38 & 0,070 & 0,38 \\
Ultrafiltrat pH 6,6 & 0,50 & 0,076 & 0,43 \\
Ultrafiltrat pH 6,0 & 0,61 & 0,078 & 0,48 \\
Ultrafiltrat pH 5,8 & 0,80 & 0,081 & 0,54 \\
Ultrafiltrat pH 5,6 & & & \\
\hline
\end{tabular}

La solubilisation observée aux $\mathrm{pH}$ acides est en bon accord avec les résultats précédemment obtenus par Davies et White (1960).

Les figures 4-5-6 indiquent que les teneurs respectives en $\mathrm{Ca}-\mathrm{Mg}-\mathrm{P}$ des rétentats d'ultrafiltration augmentent d'une manière directement proportionnelle au facteur de concentration. Si la teneur en un élément minéral donné du rétentat d'ultrafiltration est $\mathbf{M}_{\mathrm{r}}, \mathrm{M}_{\mathrm{ut}}$ la teneur en cet élément dans l'ultrafiltrat et $\mathrm{M}_{1}$ la teneur en ce même élément dans le lait et si $\mathrm{F}$ est le facteur de concentration on a :

$$
\mathrm{Mr}_{\mathrm{r}}=\mathrm{M}_{\mathrm{uf}}+\left(\mathrm{M}_{\mathrm{r}}-\mathrm{M}_{\mathrm{ur}}\right) \mathrm{F}
$$

La teneur en $\mathrm{Ca}$ du rétentat obtenu par ultrafiltration du lait à $\mathrm{pH}$ 6,6 est donc égale respectivement à 1,7 et 3,8 fois la teneur en $\mathrm{Ca}$ du lait aux facteurs de concentration 2 et 5 .

A pH 6,0 ces proportions sont respectivement égales à 1,55 et 3,3 ; à $\mathrm{pH} 5,6$, à 1,4 et 2,6 . 


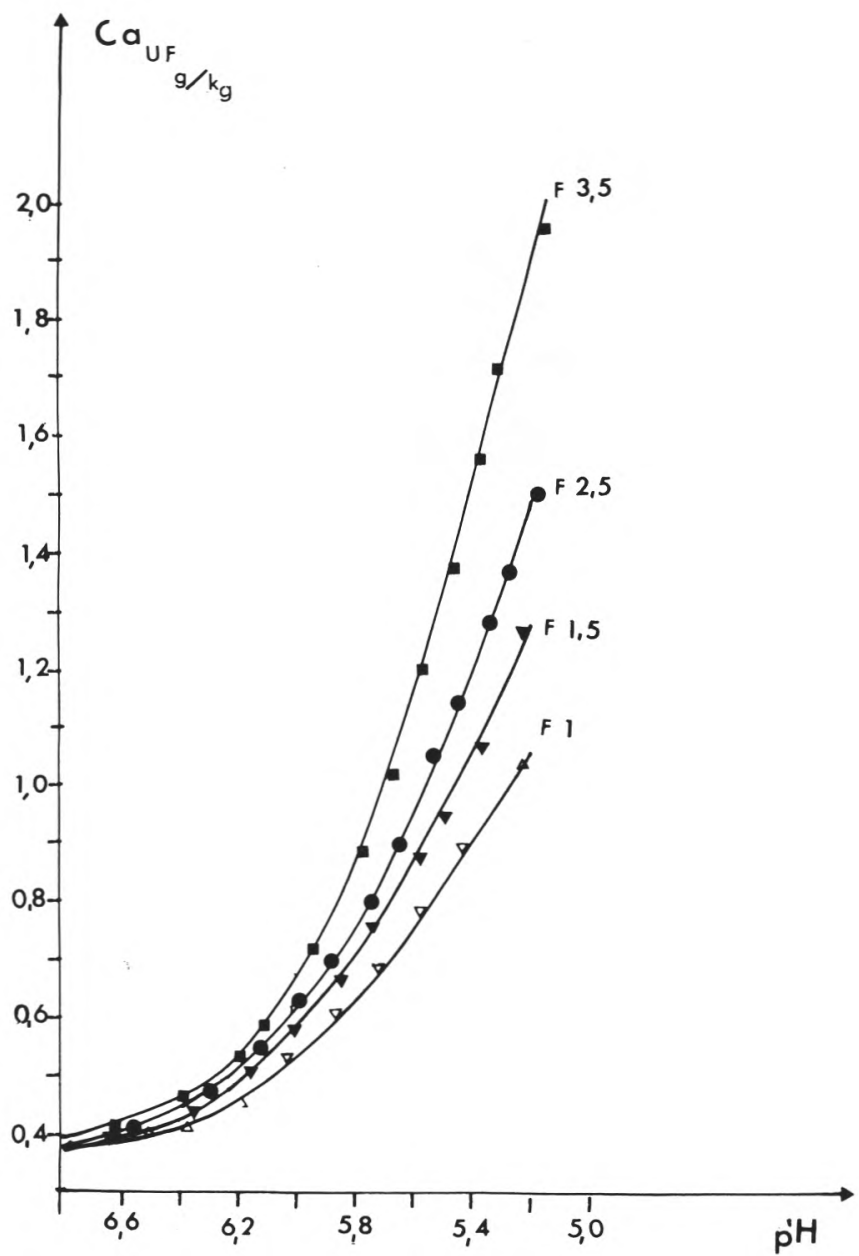

fig. 7

Variation de la teneur en calcium de l'ultrafiltrat, en fonction du $\mathrm{pH}$ à différents facteurs de concentration

Influence de l'abaissement du pH sur la composition minérale des rétentats de concentration différente

Les figures 7 - 8 et 9 montrent l'influence du $\mathrm{pH}$ sur la solubilisation du calcium, du magnésium et du phosphore du lait et des rétentats de concentrations différentes.

La quantité de minéraux présents dans l'ultrafiltrat, reflet de la fraction soluble du lait et des rétentats, est une fonction exponen- 


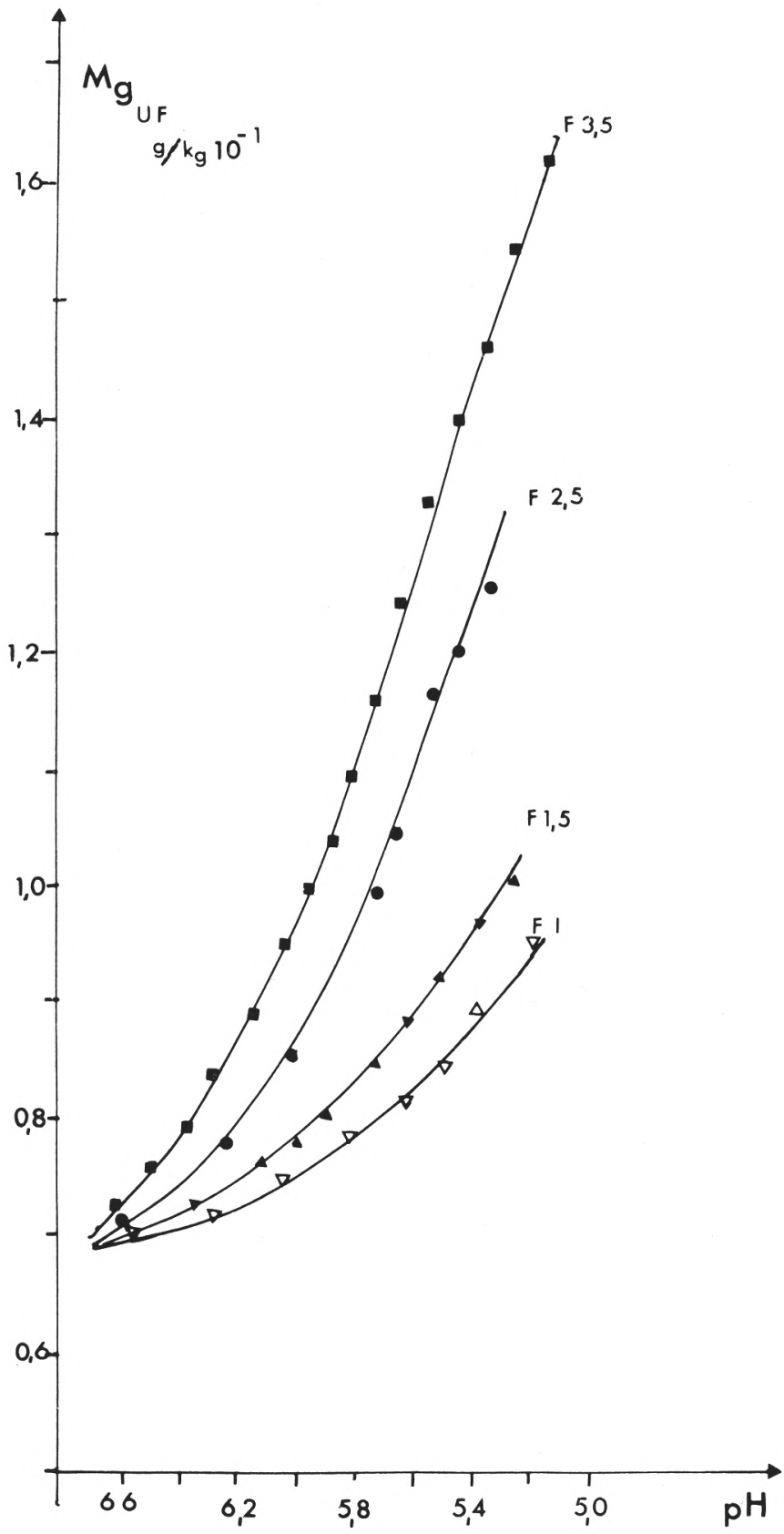

fig. 8

Variation de la teneur en magnésium de l'ultrafiltrat, en fonction du $\mathrm{pH}$ à différents facteurs de concentration 


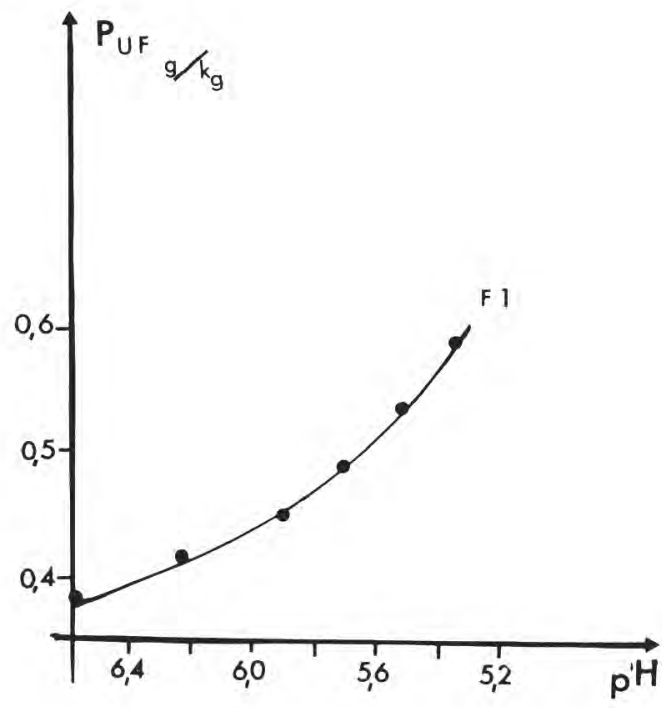

fig. 9

Teneur en phosphore de l'ultrafiltrat de lait en fonction du $\mathrm{pH}$

tielle du $\mathrm{pH}$. La quantité de minéraux solubilisés à un $\mathrm{pH}$ donné est d'autant plus importante que la teneur en élément complexé est élevée ; avant abaissement du $\mathrm{pH}$ cette teneur en élément complexé peut être aisément augmentée par ultrafiltration du lait à pH 6,6.

Les résultats obtenus montrent que quelle que soit la teneur en calcium total d'un rétentat, le rapport calcium soluble / calcium total est pratiquement constant à un pH donné. Ainsi par exemple l'ultrafiltration de lait jusqu'à un facteur de concentration de 3,5 , au $\mathrm{pH}$ du lait $(6,60)$, va nous conduire à un rétentat contenant $3,30 \mathrm{~g}$ de calcium ; l'acidification de ce rétentat va se traduire par une solubilisation de calcium dont la teneur dans le rétentat est égale au produit de la concentration en calcium de l'ultrafiltrat (fig. 7) par la quantité de phase ultrafiltrable contenue dans un $\mathrm{kg}$ de rétentat soit $\mathrm{Caut}(1-0,033 \mathrm{~F}), 0,033 \mathrm{~F}$ étant la quantité de protéines exprimée en $\mathrm{kg}$, contenue dans un $\mathrm{kg}$ de rétentat ; si ce rétentat est acidifié jusqu'à $\mathrm{pH} 5,2$, nous aurons alors environ $1,80 \mathrm{~g} / \mathrm{kg}$ de calcium soluble ce qui représente 54,5 p. 100 du calcium total. Si d'autre part nous ultrafiltrons du lait, préalablement acidifié à $\mathrm{pH} 5,2$, jusqu'à un facteur de concentration $\mathrm{F}=3,5$, nous obtenons un rétentat contenant $1,70 \mathrm{~g}$ de calcium total et $0,95 \mathrm{~g}$ de calcium soluble étant donné que Cauf $=1,05 \mathrm{~g}$, ce qui nous conduit à une proportion de 55,8 p. 100 de calcium soluble. Le pourcentage de calcium soluble par rapport au calcium total, dans un rétentat à un $\mathrm{pH}$ donné, peut être obtenu à l'aide de la formule (1) dans 
laquelle $\mathrm{Ca}_{1}$ est la teneur en calcium dans le lait et Cauf la teneur en calcium soluble de l'ultrafiltrat au $\mathrm{pH}$ étudié. Cette formule est approchée puisqu'elle ne tient pas compte de l'eau liée aux protéines, mais uniquement de la fraction azotée qui est égale à $3,3 \mathrm{~F}$ pour $100 \mathrm{~g}$ de rétentat.

$$
\frac{\mathrm{Ca} \text { soluble }}{\mathrm{Ca} \text { total }} \times 100=\frac{100-3,3 \mathrm{~F}}{\left|\frac{\mathrm{Ca}}{\mid \mathrm{Caur}}-1\right| \mathrm{F}+1}
$$

La proportion de magnésium soluble est plus élevée que celle du calcium, mais l'évolution, en fonction du $\mathrm{pH}$ et de la concentration, de la proportion de $\mathrm{Mg}$ à l'état soluble est identique à celle du calcium.

La solubilisation du phosphore en fonction du $\mathrm{pH}$ n'a été étudiée que pour le lait. On peut noter que, selon le $\mathrm{pH}$ du lait, le rapport $\frac{\mathrm{Ca}}{\mathrm{P}}$ de l'ultrafiltrat obtenu sera différent ; ainsi l'ultrafiltrat du lait à $\mathrm{pH} 6,6$, conduit à un rapport $\frac{\mathrm{Ca}}{\mathrm{P}}$ voisin de 1 dans l'ultrafiltrat. Par contre l'ultrafiltration de lait préalablement acidifié jusqu'à un $\mathrm{pH}$ de 5,2 conduit à un rapport $\frac{\mathrm{Ca}}{\mathrm{P}}$ égal à 1,7 . Les rapports $\frac{\mathrm{Ca}}{\mathrm{P}}$ des rétentats varient quant à eux non seulement en fonction du $\mathrm{pH}$ du lait soumis à l'ultrafiltration mais aussi en fonction du facteur de concentration.

\section{Pouvoir tampon des rétentats}

La figure 10 rassemble les courbes de titration effectuées sur du lait et des rétentats obtenus par ultrafiltration à pH 6,6. L'augmentation des teneurs en protéines et en minéraux des rétentats, notamment en calcium, accroît de manière très sensible leur pouvoir tampon.

L'acide lactique ajouté au rétentat se dissocie totalement en ion lactate ; en effet les protons $\mathrm{H}^{+}$sont captés par les acides organiques et minéraux dont les $\mathrm{pK}$ sont supérieurs à celui de l'acide lactique ; le déplacement de ces acides vers la forme non dissociée se traduit par une solubilisation partielle d'éléments minéraux. Ces acides organiques et minéraux responsables de l'effet tampon sont soit des eléments constitutifs des protéines (acides aminés, phosphosérine...), soit associés aux protéines (phosphate et citrate de calcium), soit soluble dans la phase aqueuse ; la concentration des protéines par ultrafiltration a donc pour conséquence l'augmentation de l'effet tampon. La quantité d'acide lactique nécessaire pour acidifier un 


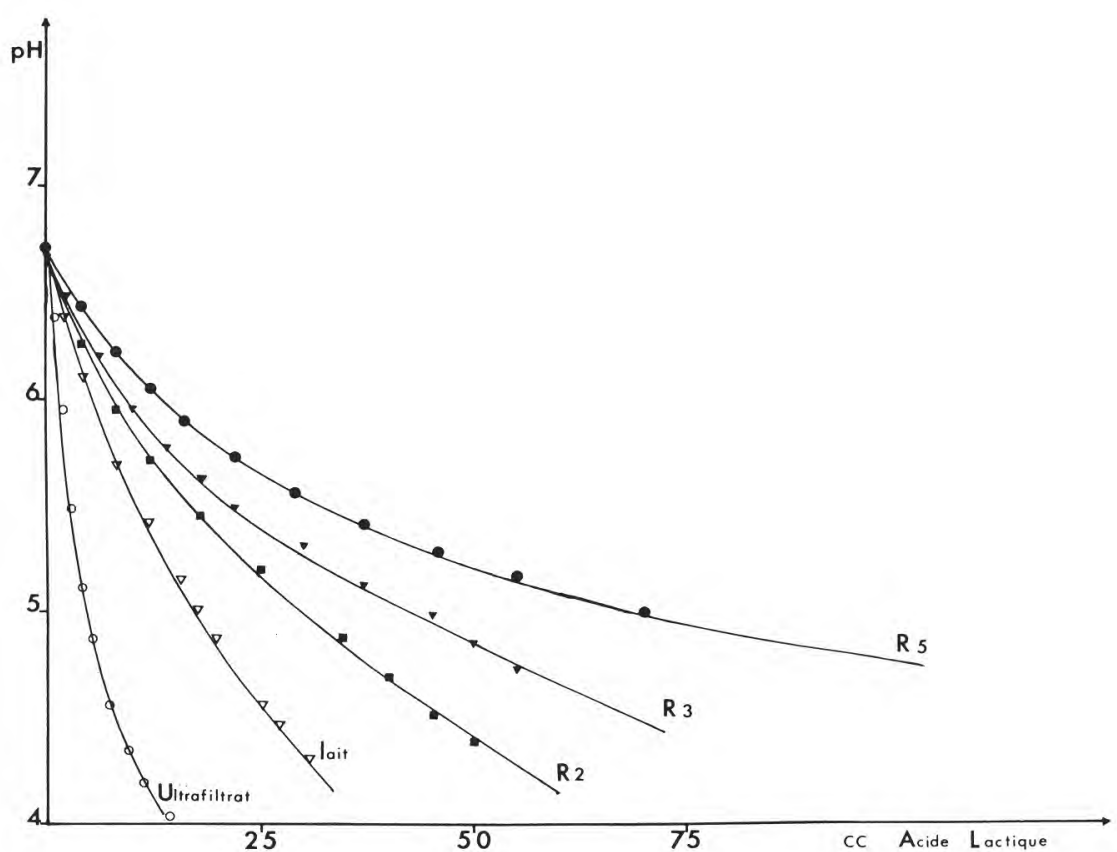

fig. 10

Variation du $\mathrm{pH}$ de $50 \mathrm{ml}$ d'ultrafiltrat, de lait et de rétentats $(\mathrm{F}=2,3,5)$ en fonction du volume d'acide lactique $0,13 \mathrm{M}$

rétentat est proportionnelle au facteur de concentration (équation 2) ; $\mathrm{A}$ et $\mathrm{B}$ sont, à un $\mathrm{pH}$ donné, les valeurs des quantités d'acide lactique nécessaire pour compenser respectivement l'effet tampon des protéines du lait et l'effet tampon de la phase ultrafiltrable. Les valeurs $A$ et $B$ peuvent être calculées d'après les courbes de titration du lait et des rétentats ; la courbe de titration de l'ultrafiltrat permet de vérifier les valeurs de $\mathrm{B}$.

$$
\mathrm{Q}_{\mathrm{L}}=\mathrm{AF}+\mathrm{B}
$$

C'est ainsi que pour acidifier un rétentat jusqu'à 4,70, il faut, par $\mathrm{kg}$ de rétentat, une quantité d'acide lactique $\mathrm{Q}_{\mathrm{L}}$ dont la valeur exprimée en $\mathrm{g}$ est donnée par l'équation (3).

$$
\mathrm{Q}_{\mathrm{L}}=4,4 \mathrm{~F}+1,5
$$

\section{Influence de l'addition de chlorure de sodium}

L'addition de chlorure de sodium au lait soumis à l'ultrafiltration sur membrane entraîne une solubilisation notable du calcium fixé aux micelles de caséine ; l'effet de cette addition sur la solubili- 


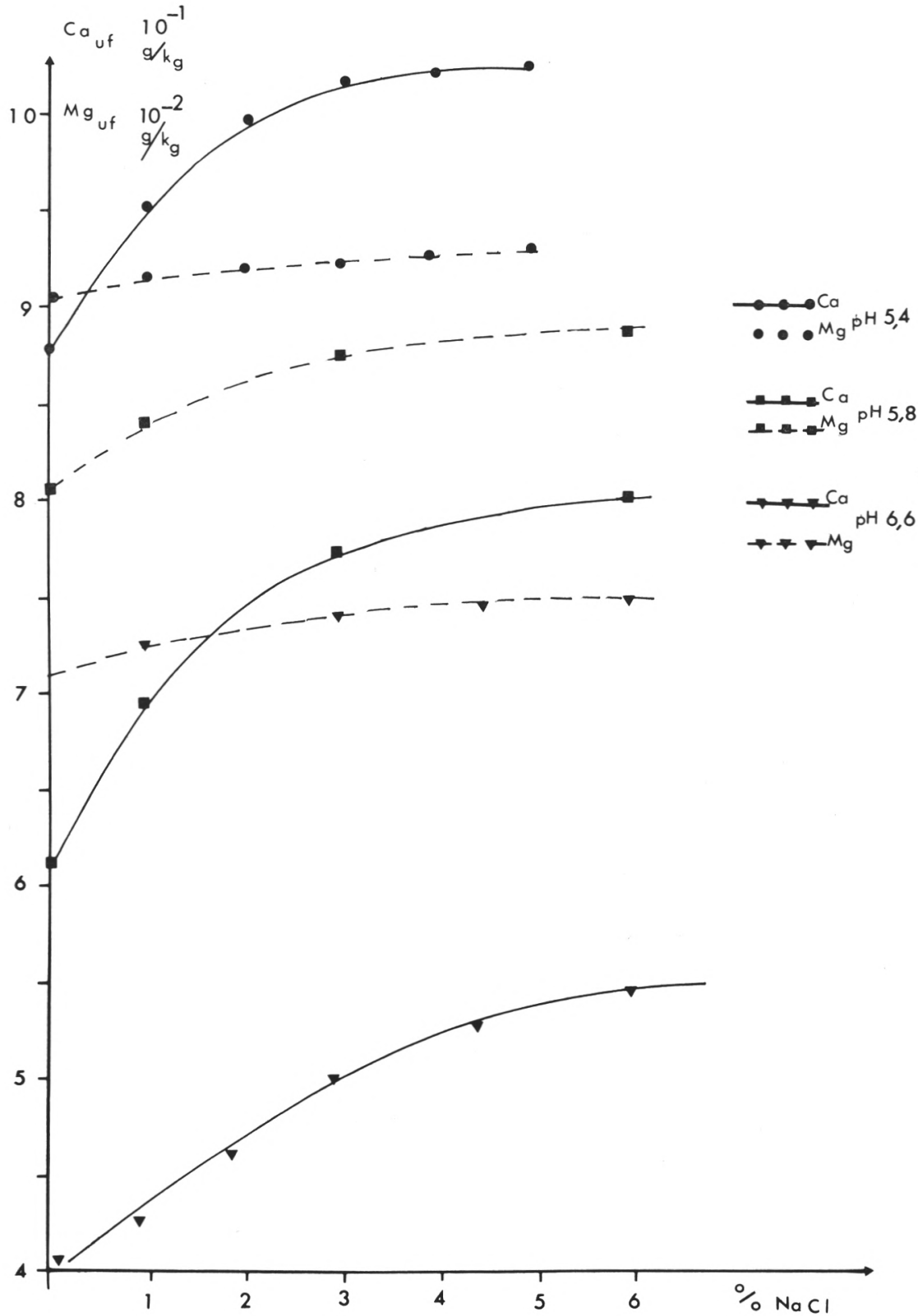

fig. 11

Variation de la teneur en calcium et magnésium d'ultrafiltrats de lait obtenus à différents $\mathrm{pH}$ en fonction de la concentration en chlorure de sodium du lait 
sation du magnésium est nettement moins prononcé. Les variations des teneurs en $\mathrm{Ca}$ et $\mathrm{Mg}$ des ultrafiltrats obtenus à partir de lait préalablement amené à différents $\mathrm{pH}$, en fonction de la quantité de sel ajoutée, sont rassemblées dans la figure 11 . Ces résultats montrent qu'une addition de 4 p. 100 de chlorure de sodium augmente la proportion de calcium soluble : à $\mathrm{pH}$ 6,6 celle-ci passe de 30 à 40 p. 100 à pH 5,8 de 50 à 62 p. 100 et à pH 5,4 de 72 à 83 p. 100. Nous n'avons pas noté de variations de la teneur en phosphore des ultrafiltrats obtenus à partir de laits préalablement additionnés de chlorure de sodium.

La solubilisation partielle du calcium et du magnésium en présence de chlorure de sodium modifie la structure micellaire des caséines ce qui se traduit par une diminution notable de l'opacité du lait ; un lait amené à $\mathrm{pH} 5,4$ et additionné de 4 p. 100 de chlorure de sodium prend l'aspect d'un lactosérum.

\section{DISCUSSION}

Les résultats, ci-dessus, montrent que le passage des minéraux au travers des membranes d'ultrafiltration est, avec les appareillages et les membranes utilisés et dans les conditions de mise en œuvre de l'ultrafiltration décrites, indépendant des caractéristiques des membranes et du type d'appareillage (conception modulaire).

Les teneurs en minéraux des ultrafiltrats correspondent aux teneurs en minéraux de la phase aqueuse du lait (Davies et White, 1960) ; elles sont par ailleurs indépendantes du facteur de concentration.

Il découle de ces résultats que :

1) dans la zone des facteurs de concentration étudiée il n'y a pas de rétention des minéraux solubles par les membranes d'ultrafiltration utilisées, donc pas d'augmentation de la teneur en minéraux dans la phase aqueuse des rétentats ;

2) il n'y a pas, au cours de l'ultrafiltration, solubilisation dans la phase aqueuse des rétentats des sels minéraux fixés aux micelles de caséine (donc la teneur des sels complexés varie proportionnellement à celle des protéines).

Les teneurs en calcium, magnésium et phosphore des ultrafiltrats et (en conséquence) des rétentats dépendent étroitement du $\mathrm{pH}$ du lait mis en ouvre et de l'évolution de ce $\mathrm{pH}$ au cours de l'ultrafiltration ; pour un rétentat donné la proportion de calcium soluble par rapport au calcium total n'est fonction que du $\mathrm{pH}$ quel que soit le mode de préparation de ce rétentat; le rapport $\frac{\mathrm{Ca}}{\mathrm{P}}$ 
d'un rétentat est variable suivant le $\mathrm{pH}$ d'ultrafiltration car, par abaissement du $\mathrm{pH}$, ces deux éléments ne se solubilisent pas dans les mêmes proportions. Grâce aux résultats acquis au cours de cette étude, il nous est possible de déterminer les conditions de préparation d'un rétentat possédant une composition minérale déterminée.

L'obtention de rétentats ayant des teneurs en protéines et en sels minéraux différentes nous a permis de mettre en évidence l'influence de la concentration sur l'effet tampon ; comme le montrent le calcul et les résultats expérimentaux, cet effet tampon est d'autant plus important que la concentration est plus élevée puisque les constituants responsables sont surtout les protéines et les sels minéraux fixés sur ces protéines.

La solubilisation du calcium et du magnésium en présence de chlorure de sodium est probablement due comme l'ont signalé Puri et Parkash (1965) et Jenness (1973) à un échange $\mathrm{Mg}^{++}$ou $\mathrm{Ca}^{++} \leftrightarrows 2 \mathrm{Na}^{+}$au niveau des micelles de caséine et non à une augmentation de la solubilité des phosphates de calcium en fonction de l'élévation de la force ionique puisque les teneurs en phosphore de la phase soluble ne varient pas. Les échanges s'effectuent sur une fraction du calcium seulement puisque nos résultats montrent que nous solubilisons au maximum 15 à 18 p. 100 du calcium micellaire ; Puri et Parkash (1965) et Jenness (1973) ont trouvé une augmentation de 20 à 27 p. 100. Le calcium solubilisé dans ce cas doit avoir des caractéristiques bien définies et différentes de celles du calcium libéré par abaissement du $\mathrm{pH}$; ces résultats tendent à confirmer les hypothèses de Manson (1962), Bohren et Wenner (1962) sur les différents types de liaison du calcium à l'intérieur de la micelle.

\section{Remerciements}

Nous tenons à exprimer nos remerciements à MM. Mocquot et Ribadeau-Dumas (I.N.R.A.) pour l'aide et les précieux conseils qu'ils nous ont apportés dans la rédaction de cette publication.

\section{R és u m é}

Les sels minéraux de la phase aqueuse du lait ne sont pas concentrés au cours de l'ultrafiltration; par contre, la teneur des éléments complexés aux protéines varie proportionnellement au facteur de concentration. Le rapport des éléments minéraux complexés et des éléments solubles des rétentats dépend uniquement du $\mathrm{pH}$ et $\mathrm{du}$ facteur de concentration. L'augmentation des teneurs en protéines et en sels a pour conséquence une élévation du pouvoir tampon ; la quantité d'acide lactique nécessaire pour atteindre un $\mathrm{pH}$ donné 
est proportionnelle au facteur de concentration. L'addition de chlorure de sodium provoque une solubilisation partielle du magnésium et du calcium.

\section{Su m m a ry}

Mineral salts of the aqueous phase of the milk aren't concentrated during ultrafiltration ; the amount of the mineral compounds bound to the milk proteins increases in the retentates proportionnally to the concentration ratio. The ratio of mineral compounds bound to proteins and soluble compounds of retentates is only a function of $\mathrm{pH}$ and concentration ratio. Buffering capacity increases with proteins and salt concentration. Lactic acid level necessary to decrease the $\mathrm{pH}$ is dependent on concentration ratio. The addition of sodium chloride to the milk leads to the solubilisation of a fraction of magnesium and calcium salts.

\section{Bibliographie}

BOHREN (H. U.) and WENNER (V, R.). - Demonstrations of calcium and phosphate interactions with casein and with synthetic ion exchanges materials. First Int. Congr. Food Sc. and Tech. 1962. Sc. publishers 1964, New-York.

Davies (D. T.) and White (J.C.D.) (1960). - The use of ultrafiltration and dialysis in isolation the aqueous phase of milk and in determining the partition of milk constituents between the aqueous and dispense phases. J. Dairy Res., 27, 171-190.

Guittonneau (G.) et Chevalier (R.) (1934). - Sur les équilibres calcophosphoriques réalisés dans les fromages. C.R. Académie des Sciences, t. 199, 801.

JENNESS (R.) (1973). - Caseins and caseinate micelles of various species. Neth. milk Dairy J., 27, 251-257.

MadSen. - Congrès de la laiterie à New-Dehli, novembre 1974.

Manson (W.) (1962). - Recent View on the structure of casein. 16 th Int. Dairy Congr. Proc., IV, 1, 513.

Maubots (J. L.) et Mocouot (G.) (1971). - Préparation de fromages à partir de " préfromage " obtenu par ultrafiltration du lait. Le Lait, 508, 495-533.

Maubois (J. L.) (1973). - Applications de l'ultrafiltration sur membrane à la fabrication de différents types de fromages. Sc, et Tech., 5, 11.

MuRthy (G. K.) et RHeH (U.) (1967). - Determination of major cations in milk by atomic absorption spectrophotometry. J. Dairy Sc., 50, 313-317.

Pompei (C.), Resmini (P.) and PeRi (C.), - Skim milk protein recovery and purification by ultrafiltration. Influence of temperature on permeation rate and retention. J. of Food Sc., 38, (5), 867-870.

PURI (B. R.) and PARKASH (1965). - Exchange of colloidal calcium with other cations in milk of three species. J. Dairy Sc., 48, 611-613.

Veisseyre (R.). - Techniques Laitières modernes 1966. Ed. "La maison rustique ", Paris. 